\title{
Filigrane
}

Écoutes psychothérapiques

\section{Miroirs de femmes}

\section{Louise Grenier}

Volume 16, numéro 1, printemps 2007

Les hauts lieux et non-lieux du rêve I

URI : https://id.erudit.org/iderudit/016180ar

DOI : https://doi.org/10.7202/016180ar

Aller au sommaire du numéro

Éditeur(s)

Revue Santé mentale au Québec

ISSN

1192-1412 (imprimé)

1911-4656 (numérique)

Découvrir la revue

Citer cet article

Grenier, L. (2007). Miroirs de femmes. Filigrane, 16(1), 99-108.

https://doi.org/10.7202/016180ar

\section{Résumé de l'article}

L'auteure résume les réflexions que lui ont inspirées les divers angles sous lesquels, à travers sa pratique clinique et ses écrits, elle a étudié la psyché féminine et ses vicissitudes. Ces angles sont : le sujet au féminin, la violence au féminin, l'Oedipe et/ou la passion au féminin ; elle illustre ces réflexions par des vignettes cliniques. d'utilisation que vous pouvez consulter en ligne.

https://apropos.erudit.org/fr/usagers/politique-dutilisation/ 


\section{Miroirs de femmes}

\section{louise grenier}

L'auteure résume les réflexions que lui ont inspirées les divers angles sous lesquels, à travers sa pratique clinique et ses écrits, elle a étudié la psyché féminine et ses vicissitudes. Ces angles sont : le sujet au féminin, la violence au féminin, l'CEdipe et/ou la passion au féminin; elle illustre ces réflexions par des vignettes cliniques.

[...] c'est une femme qui parle à d'autres femmes en leur tendant des miroirs de femmes.

Isabelle Lasvergnas, 2006, 89

Je suis ce malheureux comparable aux miroirs

Qui peuvent réfléchir mais ne peuvent pas voir

Comme eux mon œil est vide et comme eux habité

De l'absence de toi, qui fait sa cécité.

Aragon, 1963

\section{Sur fond d'absence}

ans ces miroirs de femmes que je tends à d'autres femmes, il y a des reflets de corps, de visages, de lieux et d'événements. Des images s'y démultiplient dans une sorte de mise en abîme continuelle du moi féminin. Mais les miroirs ignorent l'invisible, ils ne reflètent pas la part trouée du sexe, ses jouissances innommables, non plus que les heurts et malheurs qui fracturent la vie psychique. Dans l'imaginaire, le sexe féminin est la métaphore du vide, il est associé au trauma, à l'impensable, au rien. C'est un espace où le sujet n'est pas, disparaît, s'absente.

Sexe féminin et trauma s'évoquent mutuellement dans l'inconscient et dans les théories psychanalytiques. La découverte de la différence sexuelle est vécue - ou est théorisée - comme un événement traumatogène pour les deux sexes. Comme une violence infligée par l'autre, et qui de ce fait demeure extérieure au moi, inassimilable.

Le sexuel féminin - le sexe des mères ? - exclu réapparaît via une tonalité affective non pas de deuil mais d'attente, ce que j'appelle une expérience d'absence: absence à soi, absence à l'autre. Un événement a eu lieu, qui a percuté la psyché, l'a traversée et trouée à jamais. Pour le sujet, c'est autour de ce trou, de cette absence, que s'ordonnera désormais sa pensée du monde et de soi. 
Mon parcours clinique, mes conférences et écrits témoignent de mes rencontres au bord de ce vide central de la psyché féminine, vide traumatique et aspirant. Je raconte les attentes, passions et violences de l'Autre $^{1}$ en empruntant les voies convergentes du roman, de la biographie, du cinéma et du récit pour les faire résonner avec les histoires singulières des femmes qui se confient à moi. Je ne saurais ni penser ni écrire sans cet entrelacement continuel du littéraire et du psychanalytique dans l'écoute de l'inconscient. Ce qui est peut-être ma façon d'inviter les femmes à sortir des miroirs, à se dessaisir des images qui fomentent leur aliénation aux modes ambiantes et au dictats du discours social.

Les femmes captives des miroirs recèlent une violence par elles-mêmes ignorée. Violence subie, violence agie, responsables de certaines formes de dépression mélancolique et/ou traumatique. Dans la mélancolie, cette «autoviolence» se manifeste par une mise à mort infinie de soi, ou plutôt d'un autre incorporé au moi. Dans la dépression d'origine traumatique, cette même violence intrapsychique s'actualise par des conduites suicidaires, des dépendances toxiques, des relations passionnelles et diverses somatisations.

En parlant à d'autres femmes, en leur tendant des images de femmes, je les expulse des miroirs, je fais éclater les mirages pour en extraire les signifiants d'une histoire personnelle. En présentant des portraits de femmes souvent exceptionnelles, mais également aux prises avec des passions — féminines ? - qui entrent en conflit avec leurs quêtes narcissique et identitaire, j'amorce un mouvement de théorisation d'un sujet au féminin.

De mon parcours théorico-clinique, je retiens ici trois étapes:

- Le sujet au féminin

- La violence au féminin

- L'Edipe et/ou la passion au féminin

\section{Le sujet au féminin}

Relisant à vingt-cinq ans de distance le texte (Grenier, 2006) d'un de mes exposés sur la notion de sujet au féminin, j'y reconnais un style d'écriture et d'énonciation qui est resté le mien. J'y reconnais surtout les jalons d'une réflexion qui allait se déplier dans «Psychanalyse et féminisme»(Grenier, 1998), dans «L'étonnement du féminin» (Grenier, 2000) et dans plusieurs articles parus dans des revues de psychanalyse, notamment Filigrane.

François Peraldi animait alors un séminaire sur la notion de sujet, et il m'avait invitée à joindre ma voix à la sienne. Mon exposé reflétait mes premières lectures de Lacan de même que les critiques de Luce Irigaray (1974) sur la sexualité féminine en psychanalyse. J'y posais la question de l'avènement d'un sujet au féminin en l'articulant à celle de l'hystérie, notamment le cas Dora (Freud, 1973). De plus, j'y commentais Le ravissement de Lol V. Stein et India Song, deux romans de Marguerite Duras (1964 et 1973) où des femmes expérimentent un certain état d'absence et d'égarement traumatique sur fond d'errance sexuelle et de perte amoureuse. Paradigmes d'un exil de soi — et de la mère archaïque — d'une 
déportation du désir dans l'imaginaire (sexuel) masculin, les personnages féminins de Duras incarnent une jouissance féminine aux alentours d'un trou dans le système symbolique des représentations de la différence sexuelle. C'est une jouissance qui échappe aux impératifs phalliques et aux paramètres socioculturels qui définissent la sexualité féminine. Duras explore des territoires non cartographiées par les représentants de l'ordre sexuel. Elle traduit également une des impasses du destin féminin : l'impossible identification au père dans l'Edipe, ses absences, ses silences.

Un article de Peraldi intitulé «L'attente du père » (1984) qui avait inspiré une conférence prononcée pour la Libre association de psychanalyse de Montréal $^{2}$ ainsi qu'un dossier consacré au père dans Filigrane ${ }^{3}$ marquent le début de mon intérêt pour les effets du père absent dans la vie amoureuse féminine. Une fille sans père, ça n'existe pas, ou pour le dire autrement, impossible de se subjectiver, d'accéder à une identité sexuée féminine sans passer par le père. Non pas la personne du père, mais le père en tant que signifiant de la séparation psychologique de la mère.

C'est ce que Jean-Pierre Lebrun (2002, 52-53) éclaire remarquablement dans un article paru dans Filigrane où il dégage l'apport de Lacan par rapport à la fonction du père chez Freud. Pour ce dernier, écrit-il, la capacité de désirer passe par le renoncement à l'immédiateté du lien à la mère, ou à la jouissance de la femme-mère. La perte de cette jouissance par ailleurs impossible est introduite par le père qui par l'interdit crée un vide initial, un manque, qui pousse le sujet à chercher des objets substituts. Pour Lacan, le tiers n'est pas tant le père que le langage. Le père n'est en fait qu'un représentant de cette obligation de renoncer à la jouissance de l'autre maternel (être tout pour la mère) et d'en passer par les mots, par la chaîne du langage pour refouler l'objet du désir œdipien. En ce dernier sens, le père est d'abord un signifiant qui se substitue au désir de la mère, désir dont il permet la symbolisation. Faute de ce signifiant, et sans un père chargé de le représenter, la fille reste captive de l'autre maternel, attirée par le néant ${ }^{4}$. Faute d'un père, le duo imaginaire est sans cesse rejoué au travers des amours et séparations impossibles (Grenier, 2004 et 2006). Or, la violence est souvent l'envers tragique de ces modes fusionnels et passionnels du lien affectif.

\section{La violence au féminin}

Marguerite Duras a exploré ces régions archaïques de l'être où le féminin se dissout dans le maternel originaire, et où une femme ne rencontre dans le regard de l'autre que son absence, que son inexistence. Helga reste dans mon souvenir la personnification de cette fatalité féminine. Elle m'est apparue comme une Erynnie descendue du ciel antique.

Un jour au Sénégal, où elle vivait depuis deux ans, elle avait poignardé ses deux fils, puis avait tourné l'arme contre elle. On l'avait retrouvée délirante, son sang mêlé à celui de ses enfants. Revenue à elle, elle expliqua son geste par une jalousie meurtrière: elle avait surpris son mari au lit avec une autre femme. 
À l'hôpital psychiatrique de Fann à Dakar ${ }^{5}$, il était impossible de ne pas voir Helga. Elle circulait dans les jardins, entourée d'amis et de soignants extrêmement attentifs et bienveillants à son égard. Son crime paraissait inspirer à son entourage une sorte de respect sacré, où était-ce de l'horreur? Helga s'était transformée en déesse toute-puissante de la vengeance et par-là était devenue intouchable. Pourtant, tout dans son attitude, dans sa voix, exprimait féminité et douceur. Ses longs cheveux noirs encadraient un visage à la Greco, un corps mince et long, quasiadolescent.

Suicidaire, elle était à la fois surveillée de près et terriblement solitaire. Parfois elle m'invitait dans sa chambre où avec quelques amis nous bavardions au rythme du tam-tam. Jamais elle ne parlait de ses enfants morts, mais il ne cessait d'être présents dans son regard qui parfois s'égarait.

Je la revois au cours d'une thérapie de groupe à l'africaine. Nous, patients et visiteurs de Fann, sommes assis en cercle sous l'immense paillote en attendant que commence la séance. Helga est assise par terre aux pieds de son psychiatre levant vers lui des yeux pleins d'adoration. De l'amour, n'aura-t-elle retenu que la passion et les éclats meurtriers, me dis-je? Et qui peut vouloir encore de son amour?

La pulsion destructrice a détruit mère et enfants dans le même mouvement passionnel. Telle Médée désespérée par la trahison de Jason, Helga agit une violence qui trouve son objet dans ce qu'elle a de plus cher. Pulsion brute, primitive, transgressive qui fait sauter le verrou de la loi symbolique, et détruit tout sur son passage.

Quelques années après sa libération et être retournée en Allemagne, Helga me raconta qu'elle avait passionnément aimé le psychiatre qui l'avait sauvée de la prison et soignée. Au moment de le quitter, elle n'avait pu le lui dire. Son silence était l'effet d'une dissociation psychique face à un événement qui lui faisait violence et qui à ce titre faisait office de trauma. Figée devant l'imminence d'une perte, son amour lui était resté en travers de la gorge, de même que sa douleur. Elle savait qu'elle ne le reverrait plus jamais, et dans ce «never more», son moi disparaissait. Désormais, elle serait une femme «avec personne dedans ${ }^{6}{ }$. Un être sans objet, sans objet d'amour, précisa-t-elle. Dans son imaginaire, se télescopaient les images du mari infidèle, les traces mnésiques de l'abandon maternel et le deuil impossible de son thérapeute.

Helga avait été abandonnée à la naissance, puis confiée à un orphelinat où elle avait été négligée et maltraitée. Du point de vue infantile, la mère absente et l'institution chargée de son éducation sont des agresseurs tout-puissants. Pour survivre psychiquement, est-il possible que la petite ait incorporé une figure préhistorique de la mère définie par sa seule destructivité ? En cas de perte d'objet et/ou de rupture du lien, cette même violence de l'autre primitif redevient agissante. Or, cet «autre» ignore toute loi, toute limite, tout interdit. Il tue. Or, en tuant ses enfants, Helga avait perpétré l'impensable. Elle avait agi une violence clivée, non symbolisée, dévoilant par-là les effets destructeurs et durables du trauma narcissique primitif. 
Impossible de réduire le crime d'Helga aux effets irrépressibles d'une dépression psychotique. Son acte venait à la place d'un signifiant exclu, forclos de l'inconscient et du langage, il jetait à la face du monde une violence pulsionnelle au féminin qui dans l'imaginaire collectif est associé aux déesses maternelles, donneuses de vie et de mort. Dans sa manière d'aimer, l'autre était tout, inséparable de son moi. Il ne pouvait pas la rejeter, ni la tromper. Il ne pouvait que faire Un avec elle. Toute perte précipitait Helga dans un espace dominé par des pulsions élémentaires. Et c'était un espace sans père.

Comment ne pas évoquer ici «L'Aimée» (Lacan, 1975), cette paranoïaque poussée au meurtre par son délire, mais aussi par une sorte de perversion incestueuse dans le rapport à la mère, et dont la sœur est une représentante. Aimée est prise dans un imaginaire maternel terrifiant et persécuteur qui exclut le père dans sa fonction séparatrice. Du fait cette exclusion, elle est aliénée à un personnage féminin idéal auquel elle s'identifie pour ne pas la désirer. Plus précisément, l'identification imaginaire recouvre ici un désir homosexuel inassimilable pour le moi. Aimée serait en quelque sorte le signifiant d'une féminité sexuelle haïe et engluée dans la chose maternelle. En était-il ainsi pour Helga, planait-il sur elle l'ombre des amours impossibles?

\section{L'Cdipe et/ou la passion au féminin}

Il arrive que le drame principal d'un sujet ne soit pas tant ce qu'il a vécu, ce qui après tout peut faire l'objet d'un récit, que ce qu'il n'a pas vécu et aurait dû vivre. Dans ce cas, il est impossible de raconter l'événement, encore moins de s'en souvenir, puisque pour le sujet, il n'y a rien. Rien de tangible, rien de communicable. Ce que d'aucuns appellent un trauma négatif, le défaut d'un événement fondateur du narcissisme constituant ici l'agression (Duparc, 2001-2002, Ferenczi, 1982, 1985).

La plupart des femmes que j'écoute racontent leur vie avec moult détails et affects. Leurs récits ne sont pas si différents des Dora et Irma de Freud (Freud, 1973), de la Sabina (Spielrein, 2004) de Jung et de l'Aimée de Lacan (1973). Dans mon imaginaire, elles mettent leurs pas dans ceux de la Gradiva de Jensen ${ }^{7}$, de la Thérèse d'Arthur Schnitzler (Schnitzler, 1981), d'Adèle Hugo (Delbée, 1982) et de Camille Claudel (Smith, 1996). Cortège de femmes qui chacune à sa façon tente de trouver sa place et sa fonction dans le champ du désir, de formuler sa demande dans des voies déjà frayées par un amour infantile. Pourtant, quelque chose manque toujours au récit - une présence, un amour, un mot - qui aurait permis au sujet de se construire et d'exister. Un non-événement, une sorte de ratage déterminent la façon dont le sujet traversera l'Edipe.

Laura et Olympia s'opposent du point de vue de leur rapport à l'Edipe. La première est incapable d'en sortir, la seconde d'y entrer. Cependant, toutes deux sont marquées par un vécu traumatique. Ce vécu, forclos de la structuration œdipienne du désir sexuel, fait retour dans le réel sous forme de mouvements passionnels destructeurs et/ou de conduites autodestructrices. 


\section{Laura, fille d'CEdipe}

Triangle classique: depuis dix ans, Laura est amoureuse d'un homme marié. Dans les moments de crise, elle accuse son amant d'avoir abusé d'elle et menace de se suicider. Sa douleur s'étale alors au su et au vu de tous, incluant son mari. La relation passionnelle est ponctuée de ruptures et de retrouvailles, d'espoir et de désespoir. Elle a besoin de désirer pour se sentir vivante, mais elle ne peut désirer qu'à la manière d'un homme, c'est-à-dire depuis une identification masculine. En effet, l'homme aimé occupe dans son fantasme une position généralement considérée comme féminine, il est l'objet à conquérir, à posséder. L'amant accepte pour un temps de jouer le rôle qui lui est imparti dans le scénario mais finit toujours par se retirer et choisir de rester avec sa femme.

Les quelques rares moments d'intimité volés au quotidien ne font qu'exacerber le désir de Laura et lui redonner espoir d'une vie partagée. La bisexualité en jeu dans le mouvement passionnel, évidente pour elle et son amant, recouvre sa quête identificatoire féminine. Pour elle, il s'agit moins d'atteindre une satisfaction sexuelle que de se voir «femme» dans le regard de l'autre. Son amour s'adresse autant à cette image idéale qu'à l'homme qui en est le dépositaire. Il est le miroir où sa féminité semble captive.

Dans les miroirs ordinaires, Laura ne voit pas d'emblée une femme, mais un corps à l'allure masculine. Adolescente, elle a été anorexique pour «être comme sa mère ». C'est par l'homme aimé, par un détour identificatoire à un homme désirant une femme, c'est-à-dire elle-même, qu'elle se rejoint comme objet de désir et comme femme. Détour qui suppose de tester répétitivement le désir et la puissance virile de l'autre. Une fois la conquête réussie, elle peut abandonner son identification masculine et jouir de sa féminité. Comment ne pas reconnaître ici la Dora de Freud? Une femme qui par-delà l'hétérosexualité affichée et le conflit odipien typique s'identifie inconsciemment à une femme idéalisée. Sans cesse, Laura remet son scénario à l'épreuve du désir de l'autre aimé, et à chaque fois, c'est l'autre femme, la femme-mère, qui est choisie. Demeurer dans le mouvement répétitif de cette perte lui épargne-t-il la prise de conscience du désir qui sous-tend son identification?

Chez Laura, la sexualité féminine s'arrime à une scène dite traumatique qui fut racontée à l'enfant. Elle a dix-huit mois quand dans les bras d'une gardienne, elle regarde partir l'ambulance qui emporte son père décédé dans un accident. Dans un autre souvenir, le sien cette fois, elle voit un homme très gentil qui joue avec elle et la traite paternellement. Cet homme, elle l'adore, mais il va disparaître au bout de quelques années en laissant des traces psychiques durables.

Dans la passion œdipienne, Laura actualise inconsciemment l'événement qui a fracturé sa préhistoire: elle se retrouve au bord d'une perte impossible. Or, cette perte déjà advenue troue le miroir, troue le fantasme œdipien. C'est une souffrance sans sujet. Pour exister, Laura se fraie un chemin jusqu'à l'Edipe, reconstitue le triangle œdipien, ce qui lui permet à la fois de s'identifier aux deux sexes, de réaliser son désir incestueux et son fantasme de toute-puissance. 
«La mort m'attire», dit Laura, la mort est au bout du regard de l'homme aimé, elle l'épie derrière les miroirs. Tel ces Vanités des peintres de la Renaissance qui faisaient apparaître un crâne dans des miroirs modifiés par anamorphose. La mort était le véritable sujet du tableau, et peut-être que la mort est le véritable sujet de la passion de Laura. La mort, c'est-à-dire le père mort.

En apparence, Laura n'a rien d'une Dora ou d'une Irma du temps de Freud (Freud, 1973), elle a hérité des acquis du féminisme de la génération précédente, et s'est bien insérée aux plans social et professionnel. Pourtant, elle a conservé quelque chose de ces «belles hystériques». Elle aussi, en sa névrose, tente de symboliser un conflit identificatoire entre les pôles masculin et féminin de sa vie psychique. Elle s'entête à désirer non pas un homme, mais l'amour d'un «père » qui reconnaît son désir et sa féminité sexuelle. Qu'est-ce cela veut dire être femme ? La réponse à cette question, elle l'attend d'un homme-père avec qui dans l'imaginaire elle entretient une relation incestueuse.

\section{Olympia, le désir «d'être dedans »}

Être dans l'autre, s'y engloutir, comment est-ce possible? Au début de la quarantaine, après un divorce et plusieurs déceptions, Olympia a enfin trouvé l'amour parfait, un homme pour qui elle est tout. Pour la première fois de sa vie, elle se sent aimée absolument. Comment résister au paradis? Comment résister à un excès d'attention, d'affection, de tendresse ? La voilà propulsée au somment d'un rêve merveilleux jusqu'à ce qu'une fine craquelure ne vienne entamer cette bulle fusionnelle. Parce que lui, l'homme, ne supporte aucune autre personne, voire aucune autre pensée, entre elle et lui. Ici, le crime, c'est de ne pas faire Un.

«Suis-je folle?» me demande Olympia. Folle d'amour sans doute. Depuis quatre ans, elle est la cause et l'objet du délire de son amant. Un amant qui la manipule, la contrôle et ne veut qu'une chose, sa soumission. Il entre dans une fureur dangereuse face à toute intrusion réelle et imaginaire. Alors, cette femme perd pied, se met à genoux aux sens propre et figuré pour éviter le rejet et la perte d'amour. Après avoir été battue et injuriée de toutes les manières, elle revient en s'excusant au pied de son maître.

Olympia est incapable de se séparer non par peur des représailles mais parce qu'elle n'est plus rien sans lui. À cet égard, son évocation du boa qui s'enroule autour de son corps pour l'étouffer illustre parfaitement la nature de ce lien aussi essentiel que mortifère. Dans ce jeu pervers, où elle consent à être la chose de l'autre, elle retrouve l'infans qu'elle aura été. Cet homme la tient sous son emprise après l'avoir dépouillée de son narcissisme. Il joue à être dieu, jouit de ce pouvoir absolu sur la vie de sa maîtresse qui en retour jouit d'être tout pour son amant.

Séparée de son amant, ne serait-ce que par une querelle, Olympia sombre dans un état psychotique: prostration, stupeur, insomnie, perte d'appétit, ruminations, inertie, auto-reproches quasi délirants et obsessions de l'autre. Interrogée sur son passé, elle fait état d'une enfance marquée par l'abandon et des abus physiques et psychologiques de la part de ses parents. 
Son besoin d'amour est un gouffre, mais du sexe, ne veut rien savoir. Du sexe, ne connaît que le viol. Pour pallier ce manque, ce non-accès au désir sexuel, elle fait de son corps et de son visage une sorte de poupée animée et manipulée par l'autre. Ce qui évoque l'automate Olympia dans «L'homme au sable» des contes de Hoffman, et dont Freud fait l'analyse dans «L'inquiétante étrangeté» (Freud, 1971)

Laura est envahie au point d'en perdre la capacité de penser et de se protéger. Dans ce jeu pervers, la pulsion de mort est à l'œuvre, et d'une certaine façon agie par l'autre, pour défaire les identifications du moi et les liens affectifs du sujet. $\mathrm{Ne}$ reste plus que l'Autre $^{8}$ préhistorique investi de toute-puissance et incarné par l'homme mis en position de maître. Prise dans le vertige angoissant de se détruire ou d'être détruite, Olympia fait appel à un tiers, l'analyste, qui saura peut-être trancher la tête du boa constrictor.

Elle vient dire la honte et l'humiliation vécues dans cette relation abusive. Jusqu'où peut aller une femme pour être aimée, se demande Olympia? Jusqu'à quelle abjection peut-elle descendre pour occuper la place qui lui est assignée par l'Autre? Il semble que chez Olympia, cette place lui ait d'abord été désignée par une mère narcissique et par un père violent.

Olympia est une proche parente de ces femmes piégées dans un rapport transférentiel passionnel. Sabina Spielrein (2004), patiente amoureuse de Jung, séduite et abandonnée par lui, oscille entre vie et mort après cette aventure catastrophique pour elle. Marilyn Monroe (Schneider, 2006) passionnément aimée par son analyste, Greenson, ne pourra le quitter que par la mort. Pour ces femmes psychotiques ou blessées par la vie, l'autre est un lieu matriciel (source de vie et de mort) qu'il faut à tout prix investir. Il n'est pas une partie de soi, c'est elles qui font partie de l'Autre: engouffrées en lui. Dès lors, impossible de s'en séparer. Dans l'exclusion d'elles-mêmes, ne sont plus que les marionnettes du fantasme de l'autre. N'est-ce pas une façon de s'abîmer, de réaliser la pulsion de mort?

\section{Les noms de l'absent}

Que ce texte soit également un miroir que je tends aux femmes, nul n'en doutera? Dans ce parcours clinique, j'ai dégagé trois axes principaux :

- La question du sujet au féminin est liée à celle du père, ou plutôt à sa capacité de représenter la fonction symbolique de séparation dans le triangle œdipien. Cette «absence» du père a pour effet principal de maintenir les filles dans un lien duel fusionnel, sous la forme d'une identification primaire aux signifiants de l'autre et à ses attentes ;

- La prise du sujet dans l'imaginaire maternel associée à l'absence de symbolisation d'un événement traumatique se transposent dans des relations passionnelles destructrices;

- Dans la névrose, le désir sexuel féminin se pose à travers la quête d'un objet inaccessible alors que dans la psychose ou la dépression d'origine traumatique, la jouissance est liée à un engouffrement suicidaire dans l'Autre qui empêche tout désir. 
La sexualité des filles, des femmes, leur vie amoureuse peuvent être mises au service de l'autodestruction et de la haine de soi. Dans mes livres, Filles sans père. L'attente du père dans l'Imaginaire féminin (2004), dans Femme d'un seul homme. Les séparations impossibles (2006), mais aussi dans Le projet d'Antigone. Parcours vers la mort d'une fille d'Edipe (2005), j'ai voulu remonter aux sources de certaines passions destructrices en les mettant en rapport avec d'une part l'absence du père dans sa fonction symbolique de séparation de la mère imaginaire et d'autre part, avec certaines formes d'attachement extrême où le sujet féminin s'efface pour s'identifier à un signifiant et/ou à une représentation dans l'Autre. L'absent, c'est donc le père, mais aussi le sujet dès lors qu'il entre dans la passion amoureuse. À ces deux types d'absence, s'ajoute l'absence d'un signifiant du sexe, une carence symbolique pour nommer le féminin, et en particulier la jouissance féminine.

La cure analytique est faite d'une alternance de présence et d'absence, d'allers et retours dans le monde imaginaire des absents. L'analyste elle-même se fait absente, non seulement parce qu'elle s'installe hors de la vue du patient, mais parce qu'elle se tait et écoute la voix des absents dans l'autre. Sa fonction est d'occuper la place des fantômes, certes, mais aussi de les convoquer et de leur demander de s'identifier. Non pour les expulser mais pour les remplacer par des mots et des noms, et par là établir ou rétablir les fondations identitaires du sujet. Ainsi, l'analyste exerce la fonction séparatrice du père et tisse avec les femmes qu'elle écoute une toile de langage et de paroles qui leur permet de reconstruire leur histoire en y incluant les absents.

louise grenier

831, avenue rockland, bureau 106 outremont, qc h2v $2 \mathrm{z} 8$

grenier.louise@uqam.ca

\section{Notes}

1. Autre en soi au sens d'inconscient, autre hors de soi, au sens du monde réel et symbolique.

2. 15 novembre 2002, «L'attente du père dans l'imaginaire féminin». Conférence pour La libre association de psychanalyse de Montréal (LAPM).

3. Filigrane, Dossier «Tout sur mon père... » 1 et 2, Volume 11, numéros 1 et 2 , printemps et automne 2002, dont j'étais la rédactrice en chef invitée.

4. La pulsion de mort

5. J'effectuais alors un stage en ethnopsychiatrie.

6. Ici, je paraphrase Émile Ajar (Romain Gary), 1974, Gros-Câlin, Folio/Mercure de France, Saint-Amand, 1998, p. 13.

7. Voir S. Freud, 1971, Délire et rêves dans la «Gradiva» de Jensen, Paris, Idées/Gallimard.

8. Toujours cette figure de l'Autre préhistorique d'avant la différence des sexes et dont le sujet dépend absolument. Autre sans nom et sans visage. 


\section{8}

Filigrane, printemps 2007

\section{Références}

Aragon, L., 2002, Le Fou d'Elsa, Gallimard, coll. Blanche, Paris, 1963. (Dernière réédition Gallimard, coll. Poésie, n 376).

Delbée, A., 1982, Une femme, Presses de la Renaissance, Paris, et du film : Camille Claudel, 1988, réalisé par Bruno Nuytten.

Duparc F., 2001-2002, «Des traumatismes invisibles ou par manque de réalité »: http://www.spp.asso.fr/Main/ ConferencesEnLigne/Items/17.htm sur le site de la SPP.

Duras, M., 1962, L'après-midi de monsieur Andesmas, L'Imaginaire/Gallimard, Paris, 1996.

Duras, M., 1964, Le ravissement de Lol V. Stein, Paris, Folio/Gallimard.

Duras, M., 1973, India Song, Paris, Gallimard.

Ferenczi, S., 1927-1933, Psychanalyse, IV, Paris, Payot, 1982.

Ferenczi, S., Janvier-octobre 1932, Journal clinique, Paris, Payot, 1985.

Ferenczi, S., 1927-1933, «Confusion des langues entre les adultes et l'enfant. Le langage de la tendresse et de la passion », Euvres complètes IV, Paris, Payot, 1982, 125-138.

Freud, S., 1900, «Rêve de l'injection faite à Irma », L'interprétation des rêves, Paris, PUF, 1973, 98-112.

Freud, S., 1905, «Fragment d'une analyse d'hystérie (Dora)» dans Cinq psychanalyses, Paris, PUF, 1973, p. 1-91.

Freud, S., 1919, «L'inquiétante étrangeté» dans Essais de psychanalyse appliquée, Paris, Gallimard, 1971, p. 163210 .

Grenier, L., 1998, «Psychanalyse et féminisme. Un malentendu historique », Résonances, sous la direction de Simon Harel, Éditions Liber. Montréal, pp. 167-198.

Grenier, L., 2000, «L'étonnement du féminin », L'Étonnement, Montréal, sous la direction de Francine Belle-Isle, S.Harel et G. L. Moyal, Liber, pp. 121-142.

Grenier, L., 2004, Filles sans père. L'attente du père dans l'Imaginaire féminin, Montréal, Quebecor.

Grenier, L., 2005, Le projet d'Antigone. Parcours vers la mort d'une fille d'Edipe (en codirection avec Suzanne Tremblay), Montréal, Liber.

Grenier, L., 2006, Femme d'un seul homme. Les séparations impossibles, Montréal, Quebecor.

Grenier. L., 2006, «Qu'est-ce qu'être une femme? ou Réflexions sur la notion de sujet au féminin en psychanalyse » dans François Peraldi, Le sujet. Séminaire 1981-1982, Montréal, Liber, Coll. Voix psychanalytiques, p. 179-194.

Irigaray, L., 1974, Speculum de l'autre femme, Paris, Minuit.

Lacan, J., 1932, De la psychose paranoïaque dans ses rapports avec la personnalité, Paris, Éditions du Seuil, 1975.

Lasvergnas, I. 2006, «En dialogue avec l'ouvrage: Femme d'un seul homme. Les séparations impossibles, Louise Grenier, Montréal, Quebecor, 2006, dans Bulletin de la Société psychanalytique de Montréal, vol. 18. $\mathrm{n}^{\circ} 3$, 2006.p. 89-92.

Lebrun, J.-P., 2002, «Une nouvelle chance pour le père?», Filigrane, Volume 11, numéros 1, p. 50-65.

Peraldi, F., 1984, «L'attente du père», Études freudiennes, nº 23, 1984, 25-41.

Spielrein, S., Entre Freud et Jung, 2004, Paris, Aubier/Psychanalyse.

Schneider, M. 2006, Marilyn dernières séances, Paris, Grasset.

Schnitzler, A., 1981, Thérèse, Paris, Biblio/Calmann-Lévy.

Smith Dow, L., 1996, Adèle Hugo, Éditions d'Acadie, Moncton 